\title{
Development of a Novel Wireless Multi-Channel Stethograph System for Diagnosing Pulmonary and Cardiovascular Diseases
}

\author{
X. Zhang, B. B. Narakathu, D. Maddipatla, B.J. Bazuin and M.Z. Atashbar \\ Western Michigan University, Kalamazoo, MI USA \\ xingzhe.zhang@wmich.edu
}

\begin{abstract}
:
A multi-channel stethograph system was developed for diagnosing pulmonary and cardiovascular diseases (PCD). An array of 16 microphone sensors was used to record heart, lung and trachea (HLT) sounds of the patients. A 16-channel signal conditioning PCB was used to process the acquired HLT sounds. The HLT sounds are converted to time domain waveforms using a MATLAB ${ }^{\circledR}$ script. From among the converted HLT sounds, an algorithm was developed to analyze the trachea sounds for diagnosing symptoms of chronic obstructive pulmonary disease (COPD) by detecting abnormal respiratory patterns. The capability of the system to diagnose COPD was investigated by determining the respiration rate and respiration ratio, over a test period of 20 seconds. The results demonstrated that the developed algorithm was able to determine a respiration rate and ratio of 21 breaths/minute and 1.5 , respectively from the audio signal of a verified COPD patient.
\end{abstract}

Key words: Cardiovascular Diseases, Lung Sound, MATLAB, Multi-Channel Stethograph System, Respiratory Sound, Trachea Sound.

\section{Introduction}

Pulmonary and cardiovascular diseases (PCD), which often affect the heart, lung and blood vessels, are one of the major causes of mortality in the modern world [1]. PCDs can be identified by monitoring sounds from the heart, lung and trachea (HLT) [2]. An effective way to monitor/measure the HLT sounds noninvasively is by using acoustic transducers since they use low-power electronic instrumentation and consumes relatively less energy [3]. In the medical industry, stethoscopes have been used to obtain HLT sounds that is helpful in the diagnosis of PCDs [4]. However, this process is subject to a variety of limitations including observer variability, inadequate understanding of the basic mechanisms of production of the sounds, and the lack of adequate studies of clinical and physiologic correlations of the sounds themselves [4]. To overcome these limitations, a computerized HLT sound recording system with digital signal processing techniques is required to obtain the HLT sound information more objectively and with greater precision. This has led to research on the development of a robust and computerized system to record HLT sounds wirelessly for PCD analysis.

\section{Methods and Result}

System Development: A portable multichannel stethograph system was developed to non-invasively record the HLT sounds by employing an array of 16 microphone sensors, which are strategically placed in a foam pad holder (Fig. 1). A 16-channel signal conditioning $\mathrm{PCB}$ was used to process the acquired sounds from the sensor array. A wireless data acquisition module (NI-9025 and NI-9191) was used to acquire and wirelessly transmit the data to a Wi-Fi enabled tablet or smartphone, and is recorded using a LabVIEW program. A MATLAB $^{\circledR}$ script was also developed to convert the recorded data into audio files and plot the waveforms to analyze the HLT conditions.

Algorithm Development: From among the HLT sounds, the trachea sound is relatively louder and has better signal-to-noise ratio for processing [5]. An algorithm was developed to analyze the trachea sounds for diagnosing symptoms of chronic obstructive pulmonary disease (COPD) by detecting abnormal respiratory patterns. The algorithm involves four main stages: pre-processing, parameter extraction, parameter verification and parameter analysis. 


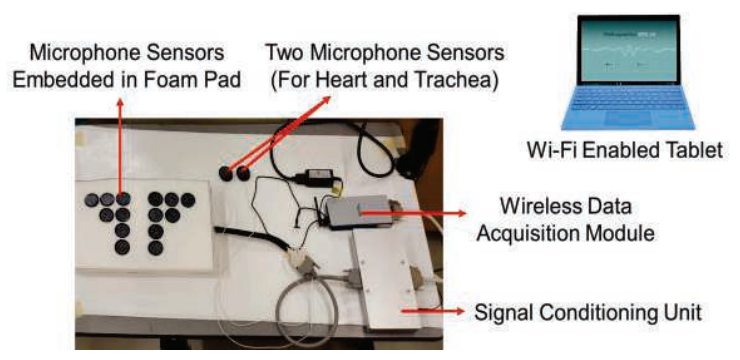

Fig. 1. Multi-channel stethograph system.

Results: The developed algorithm was employed for the analysis of an FDA verified COPD patient data, which is recorded over a duration of 20 seconds and was obtained from Stethographics, Inc. In the pre-processing stage, the acquired audio signal is converted and plotted in the time domain (Fig. 2(a)). Since the trachea sound is typically low pitched with long durations, ranging between $125 \mathrm{~Hz}$ to $500 \mathrm{~Hz}$ [6], a digital bandpass filter (cutoff frequency at $125 \mathrm{~Hz}$ and $500 \mathrm{~Hz}$ ) was used to remove white noise and unwanted signal (Fig 2(b)). The parameter extraction stage envelopes the filtered signal by using a hamming window with a $50 \%$ overlapping factor (Fig. 2(c)). In addition, a dynamic amplitude threshold, which is $25 \%$ of the total energy of the signal, is determined. Signals with amplitude that is higher than the threshold are considered to be potential breathe cycles including inhalation and exhalation sounds, and are classified as extracted parameters. The parameter verification stage involves qualification of the extracted parameters. The time duration of all the breathe cycles, including inhalation and exhalation, as well as the duration between the inhalation and exhalation of each breathe cycle is calculated using zerocrossing technique. These calculated values are compared with a series of thresholds, based on published literature [1], to qualify the breathe cycles as "valid" or "in-valid" (Fig. 2(d)). In the parameter analysis stage, the trachea sounds are quantified by mathematically calculating the respiratory rate and respiration ratio for the breathe cycles that are qualified as "valid". A respiration rate and ratio of 21 breaths/minute and 1.5 , respectively was calculated from the audio signal of the verified COPD patient. The results demonstrated that the developed system and algorithm is capable of monitoring HLT sounds as well as processing and analyzing audio signals for diagnosing the symptoms of PCDs such as COPD.

\section{Conclusion}

In this work, a multi-channel stethograph system was successfully developed for diagnosing PCDs using an array of microphone sensors. A 16-channel signal conditioning PCB
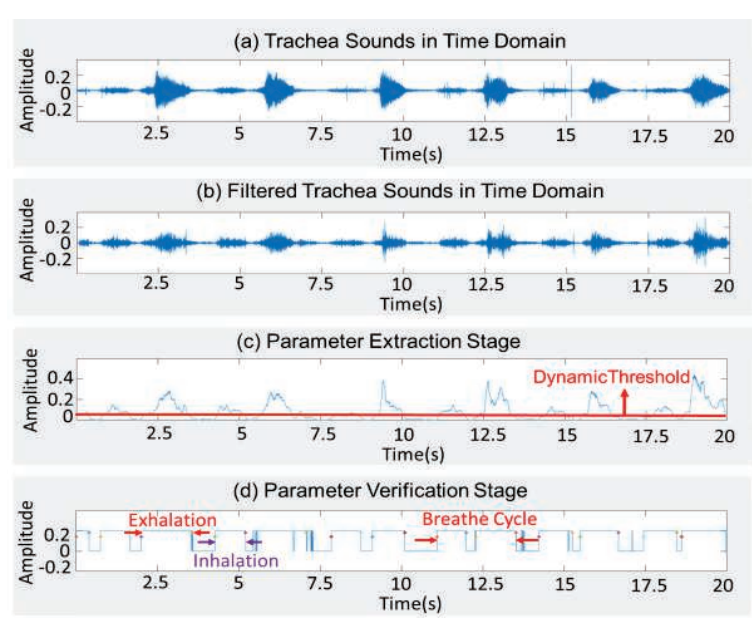

Fig. 2. (a), (b) Pre-processing stage: (c) parameter extraction stage: and (d) parameter verification stage.

along with a wireless data acquisition module was used to acquire, process and transmit the HLT sounds. An algorithm was developed, using $M A T L A B^{\circledR}$ script, to analyze the trachea sounds for diagnosing symptoms of COPD. The capability of the system to diagnose COPD was investigated by determining the respiration rate and respiration ratio, from the audio signal of a verified COPD patient over a test period of 20 seconds. The algorithm was able to determine a respiration rate and ratio of 21 breaths/minute and 1.5 , respectively thus demonstrating the capability of the system to diagnose the symptoms of COPD. Future research is underway to extend the capability of the multichannel system by developing algorithms for diagnosing other PCDs such as pneumonia, asthma, congestive heart failure (CHF) and vocal cord dysfunction(VCD).

\section{References}

[1] P. Leijdekkers, G. Valerie, Personal heart monitoring system using smart phones to detect life threatening arrhythmias, Proc. IEEE CBMS, 157-164(2006); doi: 10.1109/CBMS.2006.128.

[2] R. L. Murphy et al., Visual lung-sound characterization by time-expanded wave-form analysis, N. Engl. J. Med., 296, 968-971(1977); doi: 10.1056/NEJM197704282961704.

[3] L. Scalise, Non contact heart monitoring, Advances in electrocardiograms-methods and analysis, (2012); doi: 10.5772/22937.

[4] R. Loudon and R. L. Murphy Jr., Lung sounds, Am Rev Respir Dis, 130, 663-673(1984); doi: 10.1164/arrd.1984.130.4.663.

[5] M. Aykanat, et al, Classification of lung sounds using convolutional neural networks, EURASIP, 65(2017); doi: 10.1186/s13640-017-0213-2

[6] S. S Kraman, Speed of low-frequency sound through lungs of normal men, J Appl Physiol, 1862-1867(1983);

10.1152/jappl.1983.55.6.1862 